\section{KN Kartographische Nachrichten}

Journal of Cartography

and Geographic Information

64. Jahrgang, Oktober 2014, Heft 5

Herausgeber: Deutsche Gesellschaft für Kartographie e.V. (DGfK) - Gesellschaft für Kartographie und Geomatik

DSFK

en Gesellschaft für Kartographie e.V.

Organ der Deutschen Gesellschaft für Kartographie
(DGfK), der Schweizerischen Gesellschaft für Kartografie (SGK) und der Österreichischen Kartographischen Kommission (ÖKK) in der Österreichischen Gesellschaft für Geographie (ÖGG).

Schriftleitung

Hauptschriftleiter

Dipl.-Ing. Dr. rer. nat. h.c. Rolf Harbeck (zuständig für Aufsätze, Berichte, Ereignisse, Persönliches).

Drosselstr. 9, 53229 Bonn; rolf.harbeck@t-online.de

Fon: 0049(0)228 483593, Fax 0049(0)228 9480236

Weitere Schriftleiter

Prof. Dr. phil. Frank Dickmann (Zweiter Schriftleiter

zuständig für Hochschulnachrichten, Rezensionen).

Ruhr-Universität Bochum, Geographisches Institut,

Postfach, 44780 Bochum; frank.dickmann@rub.de

Fon: 0049(0)234 32-23379; Fax: 0049(0)234 32-14964

Dipl.-Ing. Andreas Gollenstede (zuständig für Geoinformation aktuell) Haareneschstr. 91, 26121 Oldenburg;

kn@gollenstede.com

Fon: 0049(0)441 7779-545; Fax: 0049(0)441 7779-750

Dipl.-Ing. Manfred Oster (zuständig für DGfK-, ÖKK-,

SGK-, IKV- und VKViD-Nachrichten). Zedernweg 173 B,

53757 Sankt Augustin; manfred-oster@t-online.de;

Fon: 0049(0)2241 334987

Mitarbeit für die Schweiz: Prof. Dr. Lorenz Hurni

Institut für Kartografie, ETH Hönggerberg, 8093 Zürich

hurni@karto.baug.ethz.ch

Fon: 00411 6333033; Fax: 00411631153

Mitarbeit für Österreich: Univ.-Prof. Dr. Wolfgang Kainz. Institut für Geographie und Regionalforschung,

Universität Wien, Universitätsstraße 7/5, 1010 Wien;

wolfgang.kainz@univie.ac.at

Fon: 00431 4277-8640; Fax: 00431 4277-9531

Ehrenschriftleiter

Prof. i.R. Dr. Jürgen Dodt, Witten

Editorial Board

Prof. Dr. H. Asche, Potsdam; Prof. Dr. Manfred Buchroithne Dresden; Prof. Dr. Doris Dransch, Berlin; Ass.-Prof. Dr. Sven Fuhrmann, Fairfax, Virginia, USA; Prof. Dr. Dietmar Grünreich, Ronnenberg; Prof. Dr. A. Hüttermann, Ludwigsburg;

Prof. Dr. L. Hurni, Zürich; Prof. Dr. W. Kainz, Wien; Prof. Dr.

P. Kammerer, München; Prof. Dr. W. G. Koch, Dresden; Prof Dr.-Ing. L. Meng, München; PD Dr.-Ing. J. Schoppmeyer,

Bonn; Prof. Dr. J. Schweikart, Berlin; Prof. Dr.-Ing. M. Sester

Hannover; Prof. Dr. Reinhard Zölitz, Greifswald.

Manuskripte

richten Sie bitte an den zuständigen Schriftleiter.

Hinweise zur Gestaltung des Manuskripts finden Sie unter

www.dgfk.net $>$ Publikationen $>$ KN

\section{Reviewverfahren}

Wissenschaftliche Beiträge werden im Rahmen eines

Peer-Review-Verfahrens begutachtet. Trotz sorgfältiger Redigierung übernehmen Herausgeber, Redaktion und

Verlag keine Haftung für die inhaltliche Richtigkeit der

Veröffentlichungen.

Die KN Kartographische Nachrichten ist in der internationalen Zitationsdatenbank Scopus gelistet.

Die DGfK ist Mitglied der

Internationalen Kartographischen

Vereinigung IKV/ICA

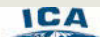

Verlag: Kirschbaum Verlag GmbH Siecriedstraße 28

D-53179 Bonn / Postfach 210209 D-53157 B B

Tel.: (0228) 95453-0. Fax: (0228)95453-27.

Internet: http://www.kirschbaum.de, E-Mail: info@kirschbaum.de

Anzeigenleiter: Volker Rutkowski; zurzeit ist Anzeigenpreisliste Nr. 49 vom 1.10.2013 gültig

Bezugspreise und ISSN: Inland 67,70 Euro jährlich, inkI. MwSt. und Versandkosten; Einzelheft 12,- Euro inkl. Mwst. Ausland 69,80 Euro jährlich, inkl. MwSt. und Versandkosten;

Einzelheft 12,- Euro inkl. Mwst.

Die Zeitschrift erscheint alle zwei Monate.

ISSN 0022-9164.

Satz: Mohr Mediendesign, Siebengebirgsweg 40

D-53773 Hennef, Internet: www.mohrmediendesign.de

Druck: SDV Saarländische Druckerei und Verlag GmbH

Werner-von-Siemens-Straße 31, D-66793 Saarwellingen

\section{Signale}

\section{Liebe Leserin, lieber Leser,}

mehr und mehr ist es in letzter Zeit Brauch geworden, ein Heft der KN einem Thema zu widmen oder es zumindest als Schwerpunktheft zu gestalten. Gründe hierfür gibt es viele: besondere Ereignisse fordern die Redaktion heraus, Vorschläge werden an die Redaktion herangetragen oder aus der Zahl der eingereichten Manuskripte bieten sich einige thematisch geradezu an, zusammen in einem Heft veröffentlicht zu werden.

Dieses Mal hätten wir gleich zwei Anlässe, dem Heft einen Namen zu geben: Zum einen ist es die INTERGEO, die vom DVW ausgerichtete international führende Kongressmesse für Geodäsie, Geoinformation und Landmanagement. Sie findet in diesem Jahr gleich zu Anfang Oktober in Berlin statt und sieht die Arbeitsplätze der Geo-Branche, zu der definitionsgemäß natürlich auch die Kartographie gehört, zwischen Erde und Cloud angesiedelt - ein schönes, treffendes Bild zum erreichten Stand der technischen Entwicklung. Um sich hierüber auszutauschen, aber auch unseren Kunden Rede und Antwort zu stehen, richten die

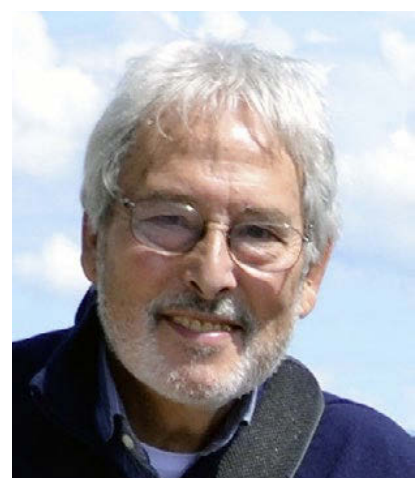
sieben Geo-Verbände wiederum den Verbändepark auf der INTERGEO aus. Auch die DGfK wird hier mit Repräsentanten des Vereins anwesend sein, um auf das breite Aufgabenspektrum der Kartographie innerhalb der Geo-Branche aufmerksam zu machen, unterstützt von einer völlig neu profilierten und gestalteten Imagebroschüre.

Zum anderen sind es der Inhalt und die Herkunft der Beiträge, die das Oktober-Heft der KN bemerkenswert machen. So widmen sich drei Beiträge disziplinübergreifenden Forschungsansätzen zu kartographischer Methodik und Wirkung, bei denen Psychologen beziehungsweise Pädagogen und nicht zuletzt Kartennutzer in hohem Maße kooperativ beteiligt sind. Aufmerksam zu machen ist auch darauf, dass drei Beiträge über Projekte unserer Nachbarn Polen und Tschechien berichten. Schon seit geraumer Zeit pflegt die KN-Redaktion die Verbindung zur Abteilung Kartographie und Geomatik an der Adam-Mickiewicz-Universität in Poznań/ Posen (Polen). In Zusammenarbeit mit Prof. Beata Medynska-Gulij konnte die Akkreditierung der KN bei der Zitationsdatenbank Copernicus International und ihre Anerkennung als Publikationsorgan durch das polnische Wissenschaftsministerium erreicht werden. Über weitere Forschungsvorhaben des innovativ arbeitenden Teams um Beata werden wir in der KN künftig wohl häufiger lesen können. Und nicht zuletzt: Auch mit Prof. Petr Kubíček vom Geographischen Institut der Masaryk-Universität Brno / Brünn (Tschechien) sind Kontakte begonnen worden, die uns neben dem vorliegenden Beitrag sicherlich weitere Berichte aus der dortigen Arbeit bringen werden.

Dies alles sind Signale, welche die KN auf dem Weg zu weiterer international-wissenschaftlicher Profilierung begleiten. Die Redaktion wertet diese Entwicklung natürlich positiv, sowohl für das künftige Redaktionsteam als auch für die DGfK, für die der bevorstehende 65. Jahrgang des von ihr herausgegebenen Journals auch weiterhin eine hohe fachliche und publizistische Verpflichtung bedeutet. Dass darüber die Ausrichtung der KN auch als Verbandsorgan mit qualifizierter Berichterstattung aus dem Vereinsleben nicht außer Acht gelassen werden darf, steht außer Frage.

Aber zurück zum Heftthema. Alle Überlegungen der Redaktion hierzu münden wohl doch in der Erkenntnis, dass die INTERGEO ein übergeordneter, vor allem einladender Anlass zu Begegnung und Austausch ist. Die KN erscheint hierzu in erhöhter Auflage, die Kartographie leistet zu Kongress und Messe ihren Beitrag. Also: eine KN zur INTERGEO 2014 in Berlin.

An Sie, liebe Leserinnen und Leser, wie immer an dieser Stelle die Empfehlung: Informieren Sie sich, unterhalten Sie sich, bereichern Sie sich - mit Ihrer KN.

Herzlich, Ihr

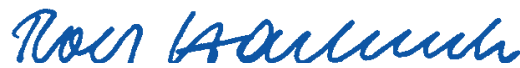

